\title{
Coautoria e participação feminina em periódicos Brasileiros da área de cirurgia: Estudo bibliométrico
}

Co-authorship and female participation in Brazilian scientific journals in the surgery field: Bibliometric study

Juliana Ravaschio Franco de Camargo ${ }^{1}$, Maria Cristina Piumbato Innocentini Hayashi ${ }^{2}$

${ }^{1}$ Universidade Estadual de Campinas

${ }^{2}$ Universidade Federal de São Carlos

\section{RESUMO}

Estudos sobre a co-autoria feminina na produção científica de várias áreas de conhecimento são frequentes na literatura científica internacional. Contudo, esse objeto de estudo tem sido pouco explorado nas pesquisas brasileiras do campo da Ciência da Informação. Esse artigo apresenta uma contribuição para essa área ao expor os resultados de uma pesquisa que investigou a coautoria feminina e a participação das mulheres no corpo editorial de periódicos científicos brasileiros da área de cirurgia publicados entre 2010 e 2014. O corpus investigado consistiu de 920 artigos publicados em quatro periódicos científicos: Acta Cirúrgica Brasileira (ACB), Arquivos Brasileiros de Cirurgia Digestiva (ABCD), Revista Brasileira de Cirurgia Cardiovascular (RBCCV) e Revista do Colégio Brasileiro de Cirurgiões (RCBC). A análise bibliométrica foi a abordagem metodológica adotada e os resultados obtidos apontaram que 920 artigos foram escritos por 5.649 coautores. Os homens aparecem como coautores em 63,5\% (n=585) dos artigos, enquanto que as mulheres comparecem como coautoras em $23,8 \%(n=219)$ do total de artigos. Ao investigar o gênero da coautoria nos artigos originais e de revisão, os resultados mostraram que a participação feminina é inferior à masculina em ambos os tipos, e em todos os quatro periódicos. Em relação à participação das mulheres nos corpos editoriais dos periódicos, os resultados revelaram que apenas no periódico ABCD a presença feminina é única e exclusiva. $\mathrm{O}$ estudo realizado demonstrou que ainda persistem as assimetrias de gênero nas coautorias, corpos editoriais e de revisores, e no comitê científico de periódicos da área de cirurgia.

PAlAVRAS-ChAVE: Co-autoria . Gênero na cirurgia.

Bibliometria. Gênero na produção científica.

\section{ABSTRACT}

Studies on female co-authorship in the scientific production of various areas of knowledge are frequent in international scientific literature. However, this object of study has been little explored by Brazilian research in the field of Information Science. This article presents a contribution to this area by presenting the results of a research that investigated female co-authorship and the participation of women in the editorial staff of Brazilian scientific journals in the area of surgery published between 2010 and 2014 . The corpus investigated consisted of 920 articles published in four scientific journals: Acta Cirúrgica Brasileira (ACB), Arquivos Brasileiros de Cirurgia Digestiva (ABCD), Revista Brasileira de Cirurgia Cardiovascular (RBCCV) and Revista do Colégio Brasileiro de Cirurgiões (RCBC). The bibliometric analysis is the methodological approach adopted and sample universe were 920 articles written by 5649 coauthors. Men appear as coauthors in $63.5 \%(n=585)$ of articles, while women show up as coauthors in $23.8 \%$ (219) of all articles. By investigating the gender of coauthorship in original and review articles, the results showed that women's participation is lower than men in both types and in all four journals. Observing the participation of women in editorial boards of the journals, the results revealed that in only one journal (ABCD) the female presence is unique and exclusive. The study showed that gender inequality persists in terms of authorship, coauthorships, types of articles, and also on the editorial board, scientific committee and board of reviewers.

KEYWORDS: Co-authorship; Bibliometrics; Gender in surgery; Gender in scientific production.

\section{Correspondência}

1 Juliana Ravaschio Franco de Camargo

Universidade Estadual de Campinas.

Campinas, SP.

Email: jcamargo@iar.unicamp.br

ORCID: http://orcid.org/0000-0003-1614-2129

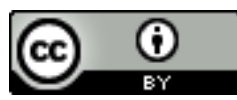

JITA: BB. Bibliometric methods. 


\section{INTRODUÇÃ̃}

$\mathrm{Na}$ literatura científica internacional a representação de gênero na ciência tem sido abordada em inúmeras pesquisas mostrando que as mulheres estão subrepresentadas na produção científica. Entre outros aspectos, estudos recentemente realizados (entre outros, por exemplo, WEST et al, 2013; EISENBERG; WHALLEY, 2015; ZENG et al, 2016) têm chamado a atenção para as maneiras sutis que as disparidades de gênero podem ocorrer na autoria e coautoria cientifica Contudo, no Brasil, pesquisas que abordem a representação feminina na autoria e coautoria ainda são escassas na área de Ciência da Informação. Para corroborar esse argumento realizamos uma rápida prospecção na biblioteca eletrônica SciELO, em dezembro de 2016, com os termos de busca "autoria" e "coautoria". Os resultados apontaram a existência de 27 artigos publicados entre 2005 e 2016, dos quais apenas um abordou a questão da participação feminina na autoria de artigos na área de neurologia, assim mesmo publicado em um periódico da área de Saúde. Entre os demais 26 artigos, apesar de onze terem sido publicados em três periódicos da área de Ciência da Informação, nenhum deles abordou a autoria e coautoria sob a perspectiva do gênero, o que justificou a realização do presente estudo, que visa, entre outros aspectos, preencher uma lacuna nos estudos métricos sob a perspectiva de gênero.

Em vista disso o estudo realizado se propôs a investigar a participação feminina na autoria, coautoria e corpo editorial de periódicos científicos brasileiros elegendo como objeto de estudo artigos publicados na área cirúrgica no período entre 2010 e 2014 da área cirúrgica. A escolha dessa área de conhecimento se deve ao fato de que embora a participação das mulheres na área da Medicina tenha aumentado durante as últimas quatro décadas, ainda persistem questões sobre a disparidade de gênero nessa área quando se trata de analisar a produção científica acadêmica desse campo de conhecimento. Inúmeras pesquisas - por exemplo, Jonasson, 2002; Jagsi et al, 2006; Morton; Sonnad, 2007; Amrein et al, 2011; Martins et al, 2012 - têm demonstrado que as mulheres ainda compõem a minoria dos autores de artigos originais de investigação, e como membros de corpos editoriais de periódicos na literatura científica da área de Medicina.

Desse modo, o estudo realizado justifica-se pela possibilidade de conhecer o perfil da produção científica em uma área específica da Medicina - a cirurgia -, e pelas contribuições que poderá oferecer para o campo dos estudos de gênero, em especial aqueles que se dedicam a compreender a participação feminina nos diferentes campos acadêmicos. Junte-se a isso, a possibilidade de contribuir com a agenda de pesquisas no campo da Ciência da Informação.

Assim, os objetivos da pesquisa foram identificar, caracterizar e analisar a produção científica brasileira consolidada em artigos científicos publicados em quatro periódicos brasileiros no período compreendido entre 2010 e 2014, em relação aos seguintes parâmetros: a) artigos e autorias: distribuição temporal dos artigos, total de autores, tipos de artigos (original ou revisão); gênero dos autores; posição dos autores (primeiro ou último) por gênero, conforme listados na assinatura dos artigos; b) periódico e corpo editorial: missão dos

\begin{tabular}{|c|c|c|c|c|c|}
\hline (C) RDBCI: Rev. Digit. Bibliotecon. Cienc. Inf. & Campinas, SP & v.15 & n.1 & p. $148-170$ & jan./abr. 2017 \\
\hline
\end{tabular}


periódicos; gênero dos editores, membros do corpo editorial, comitê científico e corpo de revisores.

O próximo tópico apresenta a fundamentação teórica que norteou a pesquisa realizada, a partir de uma revisão de literatura sobre a questão de gênero na ciência e no campo da medicina.

\section{REVISÃO DE LITERATURA}

A história da Medicina e da cirurgia no Brasil começa efetivamente com a vinda da família real. Até o início do século XIX, a licença para o exercício da Medicina não era obrigatória, conforme argumentam Moreira Junior, Figueiredo e Vieira (2012), mas os cursos que a concediam não aceitavam mulheres. Figueiredo (1999) relata que os barbeiros foram considerados os precursores dos cirurgiões, e na pesquisa que empreendeu sobre o tema, não encontrou nenhuma referência à presença de mulheres nesse ofício. Mais de dois séculos passados, esse cenário mudou radicalmente, com o aumento da presença das mulheres no exercício da Medicina e no ambiente acadêmico, embora isso não tenha sido acompanhado pela igualdade entre homens e mulheres em relação a posições de liderança, equilíbrio de salários, e eliminação de vieses de gênero. (METAXA, 2013; WEINACKER; STAPLETON, 2013). No Brasil, conforme relata Ávila (2014, p. 143), "a medicina foi majoritariamente exercida por homens até a década de 1960"; contudo, a partir de 1970 e ao longo das décadas posteriores, "as faculdades de Medicina passaram a ser um espaço de maior circulação de mulheres, aumentando mais aceleradamente na primeira década do século XXI".

No campo médico da cirurgia, a presença feminina em relação aos homens também cresceu, mas ainda está longe de se alcançar um equilíbrio entre os gêneros. (PARK et al, 2005). O estudo de Sanfey et al (2006) demonstrou que as mulheres representavam quase a metade dos estudantes de Medicina nos EUA, mas esse índice caía para quase um quarto quando a escolha das mulheres era para a carreira em cirurgia geral. Dados mais recentes compilados por Joliff,Leadley e Coakley (2012) em um relatório de avaliação sobre a participação das mulheres na Medicina acadêmica, mostram que embora a proporção de mulheres tenha aumentado ao longo dos anos entre as várias especialidades nos programas de residência médica, tais como ginecologia e obstetrícia, clínica geral e pediatria, por exemplo, em contrapartida, é menor a presença feminina em especialidades da cirurgia, como cirurgia torácica e cirurgia ortopédica. Frente a essa realidade, Harris, Chaikoff e Eidt (2007) argumentam que é necessário alterar a formação de programas treinamento de residência em Medicina.

Conforme argumenta Ávila (2014, p. 143) a "segregação ocupacional iniciada durante o processo de formação reproduz guetos masculinos e femininos no interior da profissão e limita o acesso das mulheres a especialidades e áreas de maior prestígio e remuneração". Ou seja, a feminização da Medicina, na visão da autora, provoca uma desvalorização da profissão, e a autora recorre a Bourdieu (2007), para explicar que a profissão passa a ser

\begin{tabular}{l|l|l|l|l|l|} 
(C) RDBCI: Rev. Digit. Bibliotecon. Cienc. Inf. & Campinas, SP & v.15 & n.1 & p. 148-170 & jan./abr. 2017 \\
\hline
\end{tabular}


menos valorizada, e ser vista como inferior, ao se feminizar. Ao analisar a presença das mulheres nas faculdades de medicina, Bourdieu (2007, p.108) argumentou que "a porção de mulheres decresce à medida que se sobe na hierarquia das especialidades, algumas das quais, como a cirurgia, lhes estão praticamente interditadas, ao passo que outras, como a pediatria, ou a ginecologia, lhes estão quase que reservadas".

Antes de abordar a inserção das mulheres na Medicina, vale lembrar rapidamente sua trajetória na ciência recordando alguns desafios enfrentados assim como algumas conquistas femininas nesse universo científico.

Historicamente, a ciência sempre foi apontada como uma atividade masculina. Durante os séculos XV, XVI e XVII, as mulheres eram mantidas afastadas das discussões que aconteciam nas sociedades e academias científicas; que eram consideradas as principais instituições de referência da comunidade científica mundial. Mais tarde, no século XVIII, algumas mulheres começavam ter acesso a certas tarefas consideradas de suporte da ciência; mas isso só ocorria devido à sua posição familiar, ou seja; se fossem filhas ou esposas de algum homem da ciência. De acordo com Schiebinger (2001), muitas mulheres produziam conhecimento em laboratórios montados dentro de seus lares, e os resultados desses estudos eram divulgados com nomes de seus irmãos, pais ou maridos ou algum outro representante masculino, pois aos homens era permitido produzir conhecimento científico, o que era negado às mulheres. A ausência dessa ligação com um representante masculino representava na prática a falta de oportunidade para a mulher integrar-se à comunidade científica. (HAYASHI et al, 2007). Ademais, como assinalaram Carvalho e Casagrande (2011), a ciência pode ser definida como uma construção social que se deu sob parâmetros considerados pela sociedade ocidental da época como masculinos, tais como a objetividade e a racionalidade. Dessa perspectiva, o rigor científico era considerado uma qualidade dos homens e não das mulheres.

Além desses desafios impostos para as mulheres cientistas decorrentes da sociedade patriarcal e do código androcêntrico das ciências, não se pode deixar de comentar uma questão de gênero bastante pertinente que interfere diretamente nas possibilidades das mulheres desenvolverem e produzirem conhecimentos científicos: trata-se da divisão sexual do trabalho. Quando elas deixaram suas casas e suas atividades domésticas para dedicarem-se à ciência foram criticadas e discriminadas por não estarem cumprindo seu papel de mulher estabelecido pela sociedade. A solução encontrada para lutar contra esses preconceitos foi enfrentar a dupla jornada de trabalho, e obviamente nesse aspecto as mulheres sofrem uma desvantagem, pois enquanto os homens têm tempo integral para se dedicar às atividades científicas, as mulheres, principalmente as casadas e com filhos, não possuem as mesmas chances. (CARVALHO; CASAGRANDE, 2011).

Essa condição resultou para as mulheres em um enorme acúmulo de trabalho; algo que os homens nunca enfrentaram, e as mudanças para uma divisão de trabalho mais igualitária ainda caminha a passos lentos. Mesmo com todas as dificuldades encontradas 
pelas mulheres na construção de suas carreiras científicas, vale ressaltar que elas venceram muitas barreiras e não se deixaram dominar pelos preconceitos e discriminações sofridas. Considerando o momento atual, pode-se afirmar que o passado difícil para aquelas que queriam produzir conhecimento científico já se foi, já que dados indicam que atualmente as mulheres aparecem em maior número do que os homens nas universidades. É possível afirmar então que atualmente as mulheres não sofrem mais preconceitos e discriminação na ciência?

Segundo Hayashi et al (2007), ainda persiste na sociedade, fortes elementos de machismo, de estereótipos sexuais e preconceitos contra a mulher, somados com a falta de condições e estímulos para que ela prossiga na carreira. A gravidez, por exemplo, muitas vezes é vista como um problema pelas pesquisadoras já que diversas delas não conseguem creches públicas ou mesmo privadas a preços acessíveis para obter um suporte enquanto exercem suas atividades. Devido à dedicação à família, a mulher ainda encontra dificuldades em viajar para participar de congressos que são tão importantes para o desenvolvimento em sua carreira.

Outros autores também afirmam que as mulheres ainda encontram barreiras, mesmo que quase imperceptíveis na carreira científica. Essas barreiras se tornam visíveis quando as cientistas têm de apresentar mais credenciais para obter o mesmo benefício, seja este uma promoção, uma bolsa de pesquisa ou outro tipo de vantagem acadêmica. Tal necessidade se faz notar em situações em que as mulheres são submetidas a avaliações pelos seus pares. São, portanto, vários os indícios de que, através de diversos mecanismos sutis que se estabelecem no ambiente científico, criam-se vários tipos de barreiras que dificultam a progressão profissional das mulheres. Importante apontar que muitos destes mecanismos às vezes, não são percebidos ou conscientizados pelas próprias mulheres. (OLINTO, 2011).

Olinto (2011) define então, dois tipos de mecanismos que são geralmente identificados para descrever as barreiras enfrentadas pelas mulheres: a segregação horizontal e a segregação vertical. Por meio da segregação horizontal as mulheres são levadas a fazer escolhas e seguir caminhos diferentes daqueles escolhidos ou seguidos pelos homens. Sob a influência da família e da escola, as meninas acabam se avaliando como mais aptas para o exercício de certas atividades em detrimento de outras e escolhem atividades mais compatíveis com o que consideram, ou são levados a considerar, como mais adequados para elas. A segregação horizontal inclui mecanismos que fazem com que as escolhas de carreiras sejam marcadamente segmentadas por gênero. A segregação horizontal está relacionada a outro tipo de segregação denominada como vertical, já que as profissões femininas tendem a ser menos valorizadas no mercado de trabalho.

A segregação vertical é um mecanismo social que pode ser considerado mais sutil, que tende a fazer com que as mulheres se mantenham em posições mais subordinadas, deixando de progredir em suas escolhas profissionais. Tanto a segregação vertical como a horizontal, podem sugerir uma genuína diferença de características e habilidades entre os dois 
sexos, o que explicaria a exclusão das mulheres de algumas ocupações e a sua dificuldade em atingir posições de destaque na hierarquia ocupacional. Tais características também explicariam as diferenças de gênero na academia e na atividade científica.

Analisando historicamente a relação das mulheres com os saberes produzidos pelas sociedades, e relacionando com a definição da segregação horizontal, nos deparamos com um conceito pré-estabelecido de que as mulheres são incapacitadas intelectualmente para entender as ciências mais abstratas, como a matemática, a física, a filosofia. Tal preconceito pode ser encontrado em todos os períodos da história da humanidade que considerou a mulher um ser inferior ao homem e a ele submisso. Mesmo com as modificações ocorridas na sociedade em vários aspectos e as mulheres estejam presentes em diferentes áreas de trabalho, ainda no século XXI a herança de determinados conceitos influencia tanto nas ações das mulheres quanto nos julgamentos sofridos por ela. (MOREIRA et al., 2010)

Mas alguns autores como Marinoff (2008, p.358), afirmam que desde que as mulheres conquistaram seu espaço, demonstraram rapidamente todos os tipos de excelência em ciências aplicadas e experimentais, como a Medicina e a Engenharia assim como em várias outras áreas, mostrando igualdade de competências e de desempenho nos mais altos níveis. O autor ainda comenta que a ausência histórica da mulher em contribuições nessas áreas foi um resultado de preconceitos culturais contra elas, e não qualquer déficit natural de talento ou de genialidade. Mesmo que os iluministas tenham afirmado que as mulheres tinham dificuldade para entender as ciências exatas, elas demonstraram seu talento tanto na aprendizagem quanto no ensino e desenvolvimento destas ciências.

Moreira et al. (2010) levanta uma questão interessante: sugere que ao em vez de perguntarmos "Porque tão poucas mulheres foram grandes cientistas?" poderíamos perguntar "Por que se conhece tão pouco as mulheres cientistas?" A seguir, conclui que pelas biografias das mulheres cientistas, constata-se que estas encontraram dificuldades, preconceitos, mesquinharia, e até perseguição devido ao seu sexo. Constata-se também que, proporcionalmente aos obstáculos encontrados, o número de mulheres cientistas em todas as épocas é relativamente grande e seria totalmente errôneo pensar que o progresso científico e tecnológico aconteceu sem elas. Assim, acredita que o maior dos obstáculos para as mulheres foi certamente a instrução que lhes foi negada durante séculos, já que tiveram acesso às universidades somente um século após a criação da primeira universidade.

Em relação às lideranças femininas no ambiente acadêmico, o estudo de Hayashi; Rigolin; e Hayashi (2012) apresentou os resultados de uma pesquisa que objetivou analisar a categoria "gênero" tendo como objeto de estudo projetos liderados por mulheres que participam dos Institutos Nacionais de Ciência e Tecnologia (INCTs). Os resultados mostraram, entre outros aspectos, que entre os 122 INCTs do país, apenas 18 são liderados por mulheres. 
Voltando então o olhar para a área médica e mais especificamente para a produção científica da cirurgia, que é analisada nesse artigo, torna-se interessante conhecer um pouco da inserção das mulheres na citada área, assim como conhecer algumas barreiras encontradas por elas em diversos países e que estão retratadas na literatura.

De acordo com França (2014), o papel de mulheres na "arte de curar" pode ser rastreado através do tempo, mas tradicionalmente apenas figuras masculinas são destacadas. $\mathrm{Na}$ antiguidade, as mulheres não eram admitidas nas escolas de Medicina e desta forma, a profissão médica foi considerada exclusivamente masculina. A entrada oficial do ingresso das mulheres em universidades médicas ocorreu apenas no século XIX em diversos países. No Brasil, as mulheres puderam prosseguir nessa carreira somente a partir de uma autorização de D. Pedro II, em 1879.

Com o passar do tempo, o número de mulheres na carreira médica foi aumentando gradativamente. Nos Estados Unidos, por exemplo, dados da Association of American Medical Colleges (AAMC), apontam que nos anos 1960, 90\% das candidaturas a Medicina eram do sexo masculino; nos anos 1970 e 1980 houve uma queda das candidaturas masculinas e um aumento progressivo das femininas. Em 2001, metade dos estudantes de Medicina são mulheres, tanto nos EUA quanto em outros países. (MACHADO, 2003).

O aumento do número de mulheres na área médica indica que ao longo dos anos, barreiras foram sendo derrubadas e elas foram encorajadas a progredir. As histórias das escolas de Medicina europeias demonstram a notável persistência da vontade feminina já que eram várias as dificuldades a serem enfrentadas em diversos países da Europa. Um dos exemplos que podem ser citados é a Rússia, que em 1872, admitiu 90 mulheres na Faculdade de Medicina em condições tão rigorosas e difíceis que apenas 25 terminaram o curso. Na Itália, no século $\mathrm{XV}$, as mulheres podiam concluir a graduação em Medicina, além de lecionar através de uma licença especial, mas elas não podiam observar doentes e nem fazer prescrições. (MILLER, 1994).

Em relação às especialidades da Medicina, Santos (2010) aponta que o gênero é um fator determinante da opção escolhida, pois existem áreas consideradas mais femininas e outras mais masculinas. A ginecologia, dermatologia e psiquiatria, podem ser consideradas especialidades com o perfil feminino e a cardiologia, ortopedia, proctologia e urologia, com o perfil mais masculino. Na pediatria, ocorre um equilíbrio entre os gêneros, mas na cirurgia foco dessa pesquisa - a predominância masculina sempre foi e ainda é muito acentuada. Esta área permanece como uma das que apresentam maiores obstáculos à inserção feminina, mesmo com o aumento da presença das mulheres nos últimos anos.

No Brasil, pode-se citar a história de Angelita Habr Gama, que foi a primeira mulher a fazer residência em cirurgia no país, pela Faculdade de Medicina da USP. Ela ingressou na faculdade em 1951 e no terceiro ano descobriu sua paixão pela cirurgia. Em uma entrevista concedida à Fassa (2007), Angelita lembra que a cirurgia era quase "reservada para os 
homens" e as mulheres optavam então pela pediatria, ginecologia, obstetrícia e clínica médica. Por ser uma das poucas cirurgiãs da época, Angelita passou por algumas situações inusitadas. Ao atender o paciente e depois de examiná-lo, era muito comum ouvir a seguinte indagação: "cadê o médico, quem vai me operar, que horas vai chegar o cirurgião?". A médica ainda lembra que concluiu seu pós-doutorado em Londres, aonde também foi pioneira, mas levou um tempo para ser aceita, pois só havia homens nessa especialidade.

Alguns artigos encontrados na literatura consultados para essa revisão buscaram descobrir quais os fatores que contribuem para que os estudantes de Medicina escolham determinada especialidade na residência, assim como buscam verificar a percepção das estudantes em relação às barreiras e dificuldades para atuar na área de cirurgia. Segundo Jesus (2008), existe a nível mundial, uma crise na formação quantitativa de mão-de-obra médica, especialmente entre os cirurgiões. Esse fato está relacionado à busca de melhor qualidade de vida como prioridade pelos adultos jovens. O cirurgião possui um estereótipo do profissional com dedicação irrestrita e que implica em uma vida social e familiar limitada. Os jovens modernos não desejam esse estilo de vida para si.

Embora a proporção de mulheres tenha aumentado nas escolas médicas, poucas escolhem por seguir a carreira em cirurgia geral. Um estudo realizado no Canadá aponta que apenas $21 \%$ dos residentes em cirurgia geral são mulheres e as razões que explicam esse fato, incluem a falta de modelos nos quais as mulheres possam se espelhar, as considerações sobre o estilo de vida e as percepções por parte delas, das discriminações baseadas no gênero. (PARK et al, 2005)

Em relação à falta de modelos, não fica claro se esses precisam ser do mesmo sexo para influenciar ao aluno na escolha da especialidade, mas alguns cirurgiões acreditam que estudantes do sexo feminino precisam de modelos de professores do sexo feminino e que sejam bem sucedidos. As alunas entrevistadas na pesquisa de Park et al (2005) acreditam que a carreira de cirurgiã não é compatível com uma vida familiar gratificante, um casamento feliz ou criar filhos da forma que se deseja. Além desses fatores, $25 \%$ dos estudantes do sexo feminino afirmaram ter experimentado alguma forma de discriminação devido ao gênero, advindas principalmente de pessoas da área cirúrgica. Esses fatores também se mostraram relevantes para a escolha pela carreira de cirurgia, conforme relatado nas pesquisas de Fitzgerald et al (2013) e Riska (2011).

Franco e Santos (2010) obtiveram em sua pesquisa, resultados semelhantes aos de Park et al (2005) e apontam que além de conviver com as barreiras da discriminação, falta de modelos e baixa qualidade de vida, o fator da confiabilidade e capacidade dos profissionais de cirurgia do sexo feminino aparece para desestimular o ingresso de mulheres nessa especialidade. Segundo os autores, o fenótipo masculino inspira $25 \%$ a mais de confiança do que o feminino. Isto significa que, para qualquer cargo que pleiteie, uma mulher precisa mostrar ter pelo menos, $25 \%$ mais capacidade do que seu concorrente masculino mais próximo, para ter as mesmas chances de sucesso. Além disso, características consideradas 
favoráveis para os cirurgiões, tais como personalidade forte, autocontrole, mente questionadora, capacidade de liderança e uma certa agressividade são vistas como qualidades nos homens e como componentes estranhos à personalidade feminina, gerando muitas vezes dúvidas até em relação à sua feminilidade.

Mediante todas as barreiras apontadas nos trabalhos citados anteriormente que acabam por desestimular a escolha das mulheres por essa especialidade e ciente que o número de cirurgiões do sexo feminino é consideravelmente menor em relação aos profissionais do sexo masculino, resta saber se esse panorama se reflete nas publicações científicas na área de cirurgia.

Cochran et al (2013) observam que as publicações de pesquisa nas principais revistas médicas aumentaram substancialmente ao longo das últimas quatro décadas, embora as autoras do sexo feminino continuem a ser uma minoria. De acordo com Olinto (2011), algumas evidências internacionais sugerem que há diferenças de produtividade favorecendo os homens, mas essas diferenças acabam se concentrando na fase inicial da carreira científica. As mulheres passam a mostrar mais vigor profissional que os homens em fase posterior, quando a carreira dos homens tende a se estabilizar e a delas adquire mais fôlego e tende a crescer. Assim, pode-se concluir que as diferenças de gênero na produção científica não apontam para uma clara supremacia masculina.

Já Amrein et al (2011), aponta que a autoria em pesquisas médicas possui o predomínio do sexo masculino, em diversos periódicos e várias especialidades embora haja nos últimos anos, um aumento da contribuição das mulheres. Os autores chamam a atenção para a baixa percentagem do sexo feminino como membros de conselhos editoriais, quando comparado ao número de homens envolvidos nessa atividade. Em seu estudo, objetivou-se obter uma descrição da participação das mulheres nos conselhos editoriais de 60 revistas internacionais bem qualificadas, do ano de 2011 e avaliar se há diferenças entre as revistas. Observou-se então que as mulheres são subrepresentadas nos corpos editoriais de periódicos da área médica e assim, fica claro que a desigualdade de gênero ainda existe em muitos níveis da Medicina. O autor explica que os cargos de diretoria editorial estão entre os mais altos cargos que um cientista pode alcançar em sua carreira e torna-se complicado apontar as razões para essa diferença de gênero, a não ser, se apoiar em fatores encontrados na literatura, como a existência de preconceitos implícitos e explícitos, a escassa orientação para essa atividade, a falta de modelos do sexo feminino, e, por fim, os subjetivos critérios de avaliação que colocam as mulheres em desvantagem em muitos aspectos.

No que diz respeitoàs posiçõesdo conselho editorial, Amrein et al (2011) sugerem que deveria haver mais convites para a participação de mulheres como colaboradoras dos periódicos e, se realizar essa atividade com sucesso, que elas devam ser consideradas para membros dos conselhos editoriais. Por outro lado, na sua visão, as mulheres cientistas poderiam ser mais pró-ativas e pedir para contribuir para o processo de revisão de periódicos da área médica. 


\section{MÉTODO}

A pesquisa realizada, de caráter exploratório e descritivo, utilizou como recurso metodológico a abordagem bibliométrica. Nesse tipo de estudo os fenômenos da comunicação científica - artigos, livros, capítulos de livros, por exemplo - são quantificados por meio da construção de indicadores que permitem caracterizar a produção científica a respeito de assuntos e/ou temas específicos, e desse modo compreender e analisar o estado da arte de determinada área de conhecimento. É válido mencionar, entretanto, que o conhecimento prévio do objeto de pesquisa e o contexto de sua produção, são elementos a serem levados em conta nas análises para superar os limites quantitativos presentes nesse tipo de estudo (SILVA, HAYASHI; HAYASHI, 2011).

A pesquisa elegeu como objeto de estudo artigos publicados em quatro periódicos brasileiros da área cirúrgica da medicina, e disponíveis online na biblioteca eletrônica SCIELO: Acta Cirúrgica Brasileira (ACB), Arquivos Brasileiros de Cirurgia Digestiva $(A B C D)$, Revista Brasileira de Cirurgia Cardiovascular (RBCCV) e Revista do Colégio Brasileiro de Cirurgiões $(R C B C)$. Esses periódicos foram escolhidos em função de sua representatividade, visibilidade e abrangência no campo da cirurgia, contando com sistema de "peer review" para seleção e publicação de artigos científicos, além de manterem um padrão regular e ininterrupto de suas edições, com expressiva quantidade de volumes publicados, haja vista que até a presente data, os quatro periódicos juntos já publicaram 385 números. Três desses periódicos foram criados no final dos anos 1980 - sendo que alguns títulos são continuidade de outros já existentes há quase cinquenta anos - e são editados por sociedades científicas da área (Colégio Brasileiro de Cirurgiões, Sociedade Brasileira para o Desenvolvimento da Pesquisa em Cirurgia, Colégio Brasileiro de Cirurgia Digestiva). Todos estão classificados no sistema Qualis de avaliação de periódicos da CAPES. A seleção dos artigos foi pautada nos seguintes critérios: 1) de inclusão - a) em relação ao recorte cronológico, artigos publicados no período entre 2010 e 2014, considerando que esse espaço temporal oferece uma visão atualizada da produção científica da área; b) artigos do tipo "originais" e de "revisão", por apresentarem, respectivamente, o relato completo de uma investigação - por exemplo, clínica ou experimental, no campo da cirurgia - e uma análise crítica sintética e relevante da literatura científica, a respeito de um assunto ou especialidade de interesse para a área de conhecimento focalizada; c) artigos em coautoria, pois revelam a interação e colaboração entre autores em torno de um domínio temático específico, interesses de pesquisa comuns, compartilhamento de recursos e esforços conjuntos para a busca de resultados. 2) de exclusão - a) artigos de pesquisadores chineses, pela dificuldade em identificar o gênero dos pesquisadores apenas pela grafia dos nomes, uma vez que há correlação entre pronúncia e ortografia; b) artigos publicados nos suplementos, pois além de estarem presentes em apenas dois dos quatro periódicos selecionados (ACB e ABCD), esse tipo de documento é composto por coletâneas de textos que abordam temas específicos, relatos e anais de congressos, publicados sob a forma de edição complementar ou de apoio que acompanham alguns ou todos os números do periódico; c) artigos com autoria

\begin{tabular}{|c|c|c|c|c|c|}
\hline (C) RDBCI: Rev. Digit. Bibliotecon. Cienc. Inf. & Campinas, SP & v. 15 & n. & p. $148-170$ & ian./abr. 2017 \\
\hline
\end{tabular}


exclusivamente feminina - isto é, sem autoria masculina - e artigos sem autoria feminina isto é, com autoria exclusivamente masculina, pois impedem a análise comparativa.

Além dos artigos selecionados, também compuseram o corpus de análise as informações a respeito dos corpos editoriais, de revisores e dos comitês científicos dos quatro periódicos. Com base nesses critérios a Figura 1 permite visualizar o fluxograma de seleção do corpus da pesquisa.

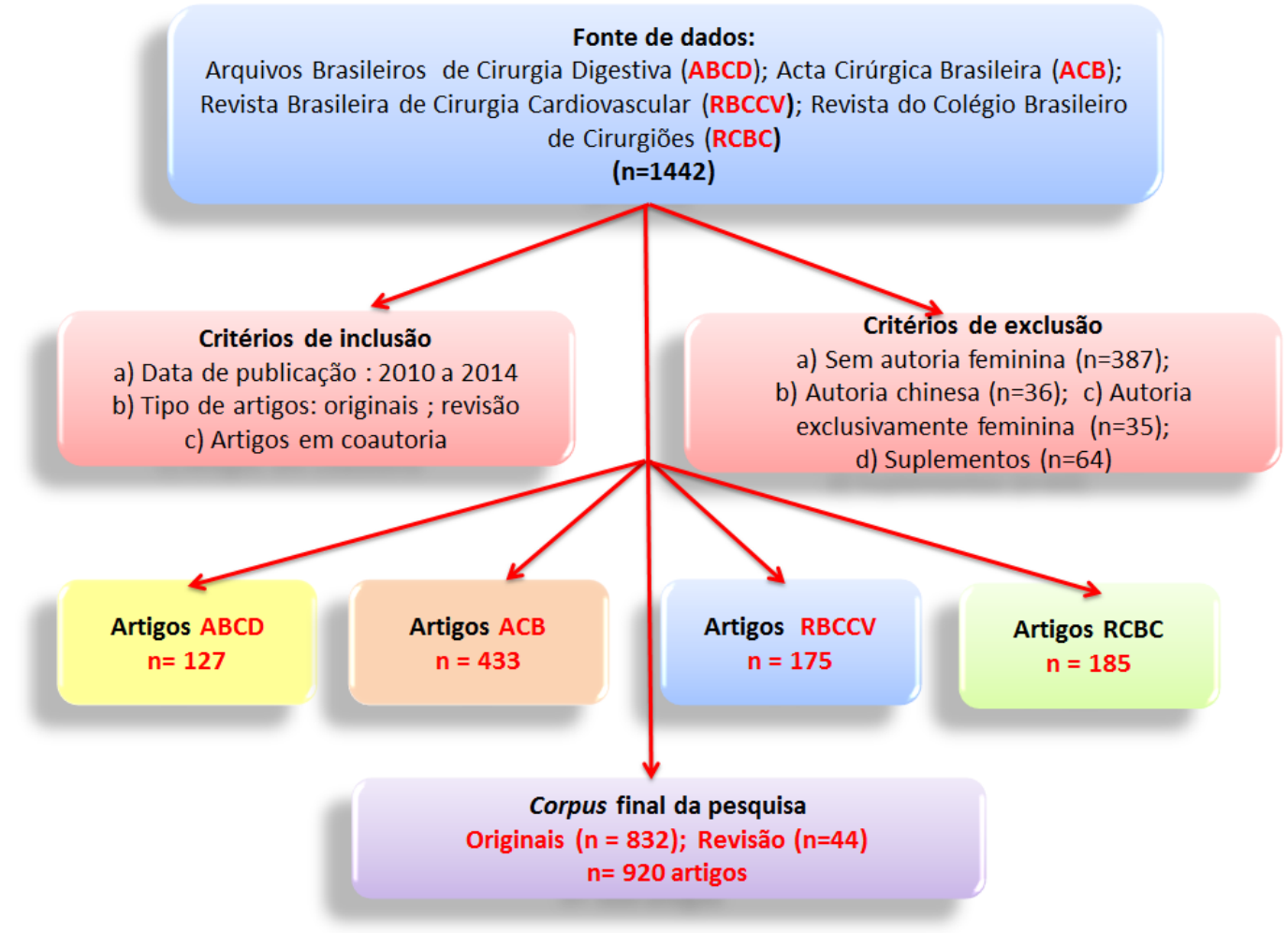

Figura 1. Fluxograma de seleção do corpus da pesquisa Fonte: Elaboração das autoras

O desenvolvimento da pesquisa foi realizado em quatro etapas, conforme descritas a seguir.

a) Etapa 1 - Revisão de literatura sobre a participação feminina em campos científicos, em particular na área de cirurgia - nessa etapa foram compulsados os principais periódicos do campo dos estudos métricos da ciência, dos estudos feministas, e da Medicina, em busca do referencial teórico sobre os aspectos de gênero presentes na medicina e que afetam a participação feminina nessa área.

b) Etapa 2 - Elaboração de protocolos para coleta e registro dos dados dos artigos e dos corpos editoriais dos periódicos - baseados no protocolo de Hayashi e Hayashi (2011) foram elaborados com o auxílio do software Excel ${ }^{\circledR}$, contendo os seguintes parâmetros bibliométricos: perfil dos artigos: título; referência completa; ano; tipo de artigo (original ou de revisão); autorias: total de autores; gênero dos autores (primeiro ou último) na assinatura 
do artigo; perfil do corpo editorial: gênero dos editores; gênero dos membros do comitê editorial, do comitê científico e do corpo de revisores.

c) Etapa 3 - Modelagem e tratamento bibliométrico dos dados - nessa fase os dados coletados foram criteriosamente conferidos, visando eliminação de inconsistências (por exemplo, registros repetidos, equívocos na categorização dos artigos) e em seguida foram transportados para o software VantagePoint ${ }^{\circledR}$ para a realização de análises bibliométricas informatizadas.

d) Etapa 4 - Análise e interpretação dos resultados - com base nos recursos teóricos e metodológicos, advindos do campo dos estudos de gênero na ciência e dos estudos bibliométricos, foram extraídos elementos que permitiram compreender as assimetrias de gênero presentes nas publicações científicas do campo da cirurgia.

Em relação aos aspectos éticos da pesquisa científica, o desenho metodológico e a natureza publicamente disponível dos dados analisados na investigação dispensaram a sua submissão a um Comitê de Ética em Pesquisa. A seguir são apresentados e discutidos os resultados obtidos.

\section{RESULTADOS}

Os 920 artigos analisados (Tabela 1) foram publicados no período entre 2010 e 2014, nos periódicos Acta Cirúrgica Brasileira (ACB), Arquivos Brasileiros de Cirurgia Digestiva (ABCD), Revista Brasileira de Cirurgia Cardiovascular (RBCCV) e Revista do Colégio Brasileiro de Cirurgiões (RCBC).

Tabela 1. Distribuição anual do corpus de análise

\begin{tabular}{|c|c|c|c|c|c|c|}
\hline \multirow{2}{*}{ Periódicos } & \multicolumn{5}{|c|}{ Anos } & \multirow[t]{2}{*}{$\begin{array}{c}\text { Total de } \\
\text { artigos }\end{array}$} \\
\hline & 2010 & 2011 & 2012 & 2013 & 2014 & \\
\hline$A B C D$ & 27 & 27 & 29 & 24 & 20 & 127 \\
\hline$A C B$ & 74 & 71 & 112 & 98 & 78 & 433 \\
\hline$R B C C V$ & 35 & 42 & 40 & 32 & 26 & 175 \\
\hline$R C B C$ & 34 & 48 & 37 & 39 & 27 & 185 \\
\hline Totais & 170 & 188 & 218 & 193 & 151 & 920 \\
\hline
\end{tabular}

Fonte: Elaboração das autoras

Os dados da Tabela 1 mostram que o periódico $A C B$ apresentou o maior número de artigos $(n=433)$, representando $47 \%$ do total analisado em relação aos demais. Quando investigamos a periodicidade desse periódico foi possível observar o crescimento do total de edições publicadas anualmente pela $A C B$. Entre 1997 (ano em que a coleção passou a ser disponibilizada na SCIELO) e 2001 foram publicados quatro edições por ano; a partir de

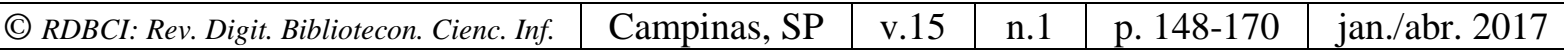


2002 até 2011, foram publicadas seis edições anuais, e de 2012 em diante a revista passou a publicar doze edições anuais.

Por sua vez, os periódicos $A B C D$ e $R B C C V$ possuem quatro edições anuais desde que foram incorporados na SCIELO (2007 e 1986), enquanto que RCBC apresenta seis edições anuais, e as edições estão disponíveis na SCIELO desde 1998. Além dessas edições, $A C B$ e $A B C D$ também publicam edições de suplementos. ABCD publicou duas edições desse tipo a partir de 2013, e $A C B$ desde 2000 já publicou 28 edições de suplementos desde 2000.

Os resultados da pesquisa também apontaram que as coautorias femininas se igualaram às masculinas em apenas 12,6\% ( $\mathrm{n}=116)$ dentre os 920 artigos analisados. Nos demais $88,3 \%$ dos artigos, as coautorias masculinas preponderam em 63,5\% $(\mathrm{n}=585)$ dos artigos. As coautorias das mulheres só foram superiores às dos homens em 23,8\% (n=219) dos artigos.

Tabela 2. Distribuição das coautorias nos artigos, por periódico e gênero

\begin{tabular}{lcccc}
\hline Periódicos & \multicolumn{3}{c}{ Artigos } & Total \\
\cline { 2 - 4 } & Mulheres & Homens & $\begin{array}{c}\text { Mulheres } \\
\text { = Homens }\end{array}$ & \\
\hline$A C B$ & 125 & 257 & 51 & 433 \\
$A B C D$ & 27 & 86 & 14 & 127 \\
$R B C C V$ & 31 & 126 & 18 & 175 \\
$R C B C$ & 36 & 116 & 33 & 185 \\
\hline Totais & $\mathbf{2 1 9}$ & $\mathbf{5 8 5}$ & $\mathbf{1 1 6}$ & $\mathbf{9 2 0}$ \\
\hline \multicolumn{5}{r}{ Fonte: Elaboração das autoras }
\end{tabular}

Também foi investigada a distribuição temporal dos dois tipos de artigos analisados. De acordo com os resultados obtidos (Tabela 3 ) houve prevalência $(95,2 \%)$ de artigos do tipo "original" (n=876) sobre os artigos de "revisão" que totalizaram 4,8\% $(n=44)$.

Tabela 3. Distribuição temporal dos artigos de revisão $(\mathrm{R})$ e originais $(\mathrm{O})$ por periódicos

\begin{tabular}{|c|c|c|c|c|c|c|c|c|c|c|c|c|}
\hline \multirow{2}{*}{ Periódicos } & \multicolumn{2}{|c|}{2014} & \multicolumn{2}{|c|}{2013} & \multicolumn{2}{|c|}{2012} & \multicolumn{2}{|c|}{2011} & \multicolumn{2}{|c|}{2010} & \multicolumn{2}{|c|}{ Subtotais } \\
\hline & $\mathbf{O}$ & $\mathbf{R}$ & $\mathbf{O}$ & $\mathbf{R}$ & $\mathbf{O}$ & $\mathbf{R}$ & $\mathbf{O}$ & $\mathbf{R}$ & $\mathbf{O}$ & $\mathbf{R}$ & $\mathbf{O}$ & $\mathbf{R}$ \\
\hline$A C B$ & 77 & 1 & 96 & 2 & 109 & 3 & 71 & 0 & 74 & 0 & 427 & 6 \\
\hline$A B C D$ & 22 & 5 & 24 & 3 & 23 & 6 & 23 & 1 & 17 & 3 & 109 & 18 \\
\hline$R B C C V$ & 23 & 3 & 31 & 1 & 38 & 2 & 37 & 5 & 34 & 1 & 163 & 12 \\
\hline$R C B C$ & 26 & 1 & 37 & 2 & 36 & 1 & 45 & 3 & 33 & 1 & 177 & 8 \\
\hline Totais & 148 & 10 & 188 & 8 & 206 & 12 & 176 & 9 & 158 & 5 & 876 & 44 \\
\hline \multicolumn{11}{|c|}{ TOTAL DE ARTIGOS } & \multicolumn{2}{|c|}{920} \\
\hline
\end{tabular}

Fonte: Elaboração das autoras

É válido notar na Tabela 3 que o periódico Acta Cirúrgica Brasileira (ACB) foi o que apresentou o menor número de "artigos de revisão" $(n=6)$, pois ao contrário dos demais não possui uma seção específica para esse tipo de artigo. De acordo com a política editorial da $A C B$, expressa nas instruções aos autores, a revista informa que só aceita artigos de revisão, e relato de caso ou de casos/trabalhos retrospectivos, quando solicitadas pelo Corpo Editorial. Isso implica que esse tipo de artigo é incluído na seção de "artigos originais". Assim, para a

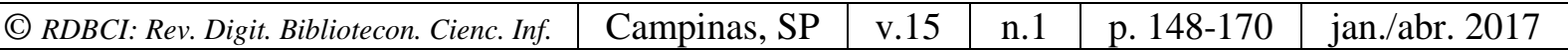


identificação de tais artigos foi realizada a leitura dos respectivos títulos e palavras-chave, e aqueles que apresentaram os termos "revisão", "metanálise", "revisão sistemática", "revisão de literatura", "revisão sistemática de literatura" foram categorizados como "artigos de revisão" e incluídos no corpus de análise.

Alguns elementos que podem servir como possíveis explicações para a preponderância dos artigos originais sobre os artigos revisão também podem ser buscados na política editorial dos quatro periódicos, conforme explicitam os textos do Quadro 1.

Quadro 1. Missão dos periódicos

\begin{tabular}{|l|l|}
\hline Periódicos & \multicolumn{1}{|c|}{ Missão } \\
\hline$A B C D$ & $\begin{array}{l}\text { Publicar artigos de estudos clínicos e experimentais que contribuam para o } \\
\text { desenvolvimento da pesquisa, ensino e assistência na área gastrenterologia cirúrgica, } \\
\text { clínica, endoscópica e outras correlatas. }\end{array}$ \\
\hline$A C B$ & $\begin{array}{l}\text { Publicar trabalhos originais de pesquisa básica e aplicada em cirurgia e ciências } \\
\text { biomédicas, novas técnicas cirúrgicas, revisões relacionadas a investigações em } \\
\text { biomedicina, artigos sobre ensino, avanços em biomedicina e comunicação científica. }\end{array}$ \\
\hline \multirow{2}{*}{$R B C C V$} & $\begin{array}{l}\text { A finalidade precípua da revista é a publicação de trabalhos representativos em cirurgia } \\
\text { cardiovascular, incluindo textos originais, pioneiros, de pesquisa, atualização, relatos de } \\
\text { caso e também a experiência apresentada no Congresso Nacional de Cirurgia Cardíaca. }\end{array}$ \\
\hline$R C B C$ & $\begin{array}{l}\text { A Revista do Colégio Brasileiro de Cirurgiões, órgão oficial do CBC, é publicada } \\
\text { bimestralmente em um único volume anual, e se propõe à divulgação de artigos de todas } \\
\text { as especialidades cirúrgicas, que contribuam para o seu ensino, desenvolvimento e } \\
\text { integração nacional. }\end{array}$ \\
\hline
\end{tabular}

Fonte: Elaboração das autoras

Os resultados obtidos também mostraram que dentre os 920 artigos analisados, 3,6\% (n=206) das coautorias eram referentes a artigos de revisão, enquanto que os artigos originais reuniram 96,4\% (5.443) das coautorias. No entanto, quando se investigou o gênero da autoria nesses dois tipos de artigos, os resultados (Tabela 4) mostraram que a participação feminina é inferior à masculina em ambos os tipos, e em todos os quatro periódicos.

Tabela 4. Distribuição das autorias por gênero nos artigos de revisão e originais

\begin{tabular}{l|cc|c|c|c}
\hline \multirow{2}{*}{ Periódicos } & \multicolumn{2}{c|}{$\begin{array}{c}\text { Coautorias em } \\
\text { Artigos de Revisão }\end{array}$} & \multicolumn{2}{c|}{$\begin{array}{c}\text { Coautorias em } \\
\text { Artigos Originais }\end{array}$} & \multirow{2}{*}{$\begin{array}{c}\text { Total de } \\
\text { coautores }\end{array}$} \\
\cline { 2 - 5 } & Homens & Mulheres & Homens & Mulheres & \\
\hline ABCD & 53 & 37 & 428 & 225 & 743 \\
\hline RBCCV & 33 & 27 & 713 & 368 & 1.141 \\
\hline ACB & 18 & 11 & 1.555 & 1.139 & 2.723 \\
\hline RCBC & 14 & 13 & 614 & 401 & 1.042 \\
\hline Subtotais & $\mathbf{1 1 8}$ & $\mathbf{8 8}$ & $\mathbf{3 . 3 1 0}$ & $\mathbf{2 . 1 3 3}$ & - \\
\hline $\begin{array}{c}(57,2 \%) \\
\text { Total Geral } \\
\text { VA/VR }\end{array}$ & $\mathbf{2 0 6}(3,6 \%)$ & $(60,8 \%)$ & $(39,2 \%)$ & \multirow{2}{*}{$\mathbf{5 . 4 4 3}(96,4 \%)$} & $\mathbf{5 . 6 4 9}(100 \%)$ \\
\hline
\end{tabular}

Fonte: Elaboração das autoras

Ou seja, os dados da Tabela 4 mostram que do total de coautores ( $\mathrm{n}=5.649$ ), apenas 42,7\% $(n=88)$ das autorias nos artigos de revisão são femininas, enquanto que em 57,2\% (n=118) as 
autorias são masculinas. Essa diferença é maior no periódico $A B C D$, em que $25,7 \%(\mathrm{n}=53)$ das autorias são masculinas e 17,9\% ( $\mathrm{n}=37)$ femininas.

Esses resultados demonstram que mesmo em artigos de revisão, realizados por meio de pesquisa bibliográfica - e que não se trata de uma pesquisa aplicada no campo da cirurgia, por exemplo - a autoria feminina também é inferior à masculina. Essa assimetria também foi observada nos artigos originais - relatos de estudos clínicos ou experimentais - publicados nos quatro periódicos, em que houve maior prevalência $(60,8 \%)$ da autoria masculina $(\mathrm{n}=3.310)$ em relação aos $39,2 \%$ da autoria feminina $(\mathrm{n}=2.133)$. Entre os quatro periódicos, $A C B$ apresentou a maior diferença $(\mathrm{n}=416)$ de autorias masculinas $(\mathrm{n}=1.555)$ em relação às femininas $(n=1.139)$ nos artigos originais. Por sua vez, RCBC apresentou a menor diferença $(n=1)$ de autorias masculinas $(n=14)$ em relação às femininas $(n=13)$.

Em relação às coautorias, os resultados da pesquisa revelaram que os 920 artigos escritos em coautoria variaram de dois até 23 coautores.

Podemos observar na Tabela 5 que os artigos com seis coautores foram o mais frequentes $(n=263)$, seguidos por aqueles com oito coautores ( $n=128$ artigos), sete coautores $(n=131)$ e cinco coautores $(n=155)$. Por sua vez, artigos com coautorias a partir de dez foram menos frequentes, variando o total de artigos de um até 12

Tabela 5. Total de artigos por periódico, coautorias por artigo e autores

\begin{tabular}{|c|c|c|c|c|c|c|}
\hline \multicolumn{4}{|c|}{ Total de Artigos } & \multirow{2}{*}{$\begin{array}{c}\text { Subtotais } \\
\text { Artigos }\end{array}$} & \multirow{2}{*}{$\begin{array}{c}\text { Total de } \\
\text { Coautores }\end{array}$} & \multirow{2}{*}{$\begin{array}{l}\text { Total de } \\
\text { autores }\end{array}$} \\
\hline ABCD & ACB & RBCCV & RCBD & & & \\
\hline 4 & 4 & 4 & 4 & 16 & 2 & 32 \\
\hline 7 & 14 & 10 & 18 & 49 & 3 & 147 \\
\hline 14 & 51 & 20 & 17 & 102 & 4 & 408 \\
\hline 19 & 81 & 20 & 35 & 155 & 5 & 775 \\
\hline 50 & 117 & 27 & 69 & 263 & 6 & 1.578 \\
\hline 24 & 64 & 19 & 24 & 131 & 7 & 917 \\
\hline 5 & 50 & 61 & 12 & 128 & 8 & 1.024 \\
\hline 1 & 29 & 8 & 4 & 42 & 9 & 378 \\
\hline 0 & 9 & 2 & 1 & 12 & 10 & 120 \\
\hline 1 & 10 & 2 & 0 & 13 & 11 & 143 \\
\hline 1 & 1 & 0 & 0 & 2 & 12 & 24 \\
\hline 0 & 2 & 2 & 1 & 5 & 13 & 65 \\
\hline 0 & 1 & 0 & 0 & 1 & 15 & 15 \\
\hline 1 & 0 & 0 & 0 & 1 & 23 & 23 \\
\hline 127 & 433 & 175 & 185 & 920 & - & 5.649 \\
\hline
\end{tabular}

Fonte: Elaboração das autoras

Outro aspecto analisado foi o gênero nas coautorias dos artigos. Os dados da Tabela 6 apontam que a coautoria feminina é inferior à masculina em todos os periódicos. 
Tabela 6. Distribuição das coautorias por gênero nos periódicos

\begin{tabular}{|c|c|c|c|c|c|c|c|c|}
\hline \multirow{3}{*}{ Coautorias } & \multicolumn{8}{|c|}{ Autores } \\
\hline & \multicolumn{2}{|c|}{ ABCD } & \multicolumn{2}{|c|}{ ACB } & \multicolumn{2}{|c|}{ RBCCV } & \multicolumn{2}{|c|}{ RCBC } \\
\hline & $\mathbf{H}$ & $\mathbf{M}$ & $\mathbf{H}$ & $\mathbf{M}$ & $\mathbf{H}$ & $\mathbf{M}$ & $\mathbf{H}$ & $\mathbf{M}$ \\
\hline 2 & 4 & 4 & 4 & 4 & 4 & 4 & 4 & 4 \\
\hline 3 & 12 & 9 & 21 & 21 & 18 & 12 & 29 & 25 \\
\hline 4 & 31 & 25 & 110 & 94 & 49 & 31 & 36 & 32 \\
\hline 5 & 59 & 36 & 225 & 180 & 62 & 38 & 109 & 66 \\
\hline 6 & 186 & 114 & 440 & 262 & 91 & 71 & 249 & 165 \\
\hline 7 & 115 & 53 & 269 & 179 & 70 & 63 & 106 & 62 \\
\hline 8 & 27 & 13 & 224 & 176 & 352 & 136 & 59 & 37 \\
\hline 9 & 8 & 1 & 131 & 130 & 55 & 17 & 19 & 17 \\
\hline 10 & 0 & 0 & 59 & 31 & 15 & 5 & 8 & 2 \\
\hline 11 & 10 & 1 & 65 & 45 & 15 & 7 & 0 & 0 \\
\hline 12 & 7 & 5 & 7 & 5 & 0 & 0 & 0 & 0 \\
\hline 13 & 0 & 0 & 11 & 15 & 15 & 11 & 9 & 4 \\
\hline 15 & 0 & 0 & 7 & 8 & 0 & 0 & 0 & 0 \\
\hline 23 & 22 & 1 & 0 & 0 & 0 & 0 & 0 & 0 \\
\hline \multirow{2}{*}{ Totais } & 481 & 262 & 1.573 & 1.150 & 746 & 395 & 628 & 414 \\
\hline & \multicolumn{8}{|c|}{5.649} \\
\hline
\end{tabular}

Fonte: Elaboração das autoras

Alguns destaques na Tabela 6 mostram a disparidade de gênero entre homens e mulheres nas coautorias. Por exemplo, no periódico $A B C D$, foi publicado um artigo com 23 coautores, dos quais 22 são homens. Esse artigo (MALAFAIA et al, 2012) apresenta diretrizes para a prevenção do tromboembolismo na cirurgia do câncer do aparelho digestivo, e como mencionaram Joliff, Leadley e Coakley (2012) a presença feminina no campo da cirurgia torácica é muito pequena. No Brasil, segundo dados das pesquisas de Franco e Santos (2010, p. 75), as cirurgias torácicas e cardíacas "possuem um pequeno contingente de especialistas do sexo feminino". Ávila (2014, p. 144) corrobora essa visão ao argumentar que "a presença feminina é muito limitada em áreas predominantemente masculinas, em especial nas cirúrgicas que utilizam tecnologia avançada", isto é, essas são áreas "de maior prestígio, e precisamente nelas, as mulheres estão em menor número".

Por sua vez, quando os artigos foram assinados por dois coautores, a igualdade de gênero foi notada em todos os periódicos. Não encontramos na literatura científica explicações para esse achado, e nem mesmo pesquisas que tivessem encontrado os mesmos resultados, permanecendo essa questão em aberto para ser investigada com mais profundidade, talvez a partir de um delineamento metodológico que incluísse a consulta aos autores dos artigos.

A pesquisa também investigou a posição da mulher na lista de assinaturas dos artigos e os resultados obtidos podem ser visualizados na Tabela 7.

\begin{tabular}{|c|c|c|c|c|c|}
\hline (C) RDBCI: Rev. Digit. Bibliotecon. Cienc. Inf. & Campinas, SP & v. 15 & n.1 & p. $148-170$ & jan./abr. 2017 \\
\hline
\end{tabular}


Tabela 7. Posição dos autores por gênero na assinatura dos artigos

\begin{tabular}{l|c|c|c|c|c}
\hline \multirow{2}{*}{ Periódico } & \multirow{2}{*}{$\begin{array}{c}\text { Total de } \\
\text { artigos }\end{array}$} & \multicolumn{2}{c|}{ Primeira Autora } & \multicolumn{2}{c}{ Última Autora } \\
\cline { 3 - 6 } & 127 & 54 & 73 & 34 & 93 \\
\hline $\mathrm{ABCD}$ & 175 & 67 & 108 & 43 & 132 \\
\hline $\mathrm{RBCCV}$ & 433 & 195 & 238 & 145 & 288 \\
\hline $\mathrm{ABC}$ & 185 & 64 & 121 & 54 & 131 \\
\hline RCBC & 920 & 380 & 540 & 276 & 644 \\
\hline Totais & \multicolumn{2}{c}{ Fonte: Elaboração das autoras }
\end{tabular}

Novamente, a assimetria de gêneros está presente, pois as mulheres não são maioria como primeiras ou últimas autoras em todos os artigos publicados nos quatro periódicos. Esses achados são corroborados, por exemplo, com aqueles encontrados nas pesquisas de Jagsi et al (2006) e Cochran et al (2013). Essas pesquisas referem que apesar do número de mulheres como primeira ou última autora terem aumentado nas últimas décadas em artigos originais nas principais revistas médicas, autoras do sexo feminino continuam a ser uma minoria substancial. Como referem Kosmulski (2012) não há um método geralmente aceito para determinar e avaliar objetivamente a contribuição de cada autor nos artigos científicos, embora haja uma tendência de que os pesquisadores sêniores ou líderes ocupem a primeira ou última posição.

A participação feminina foi investigada nos corpos editoriais dos quatro periódicos e os resultados obtidos podem ser visualizados na Tabela 8 .

Tabela 8. Participação de homens e mulheres no corpo editorial dos periódicos

\begin{tabular}{cccc}
\hline Periódico & Total de editores & Mulheres & Homens \\
\hline$A B C D$ & 1 & 1 & 0 \\
\hline$A C B$ & 9 & 2 & 7 \\
\hline$R B C C V$ & 13 & 1 & 12 \\
\hline$R C B C$ & 5 & 0 & 5 \\
\hline Total & $\mathbf{2 8}$ & $\mathbf{4}$ & $\mathbf{2 4}$ \\
\hline & Fonte: Elaboração das autoras
\end{tabular}

Os dados da Tabela 8 revelam que apenas no periódico ABCD a presença feminina é única e exclusiva, enquanto que no periódico RCBC essa situação se inverte, isto é, não há mulheres no corpo editorial. Por sua vez, a presença das mulheres é inferior nos periódicos $\operatorname{ACB}$ ( $\mathrm{n}=2$ mulheres, para $\mathrm{n}=7$ homens) e RBCCV ( $\mathrm{n}=1$ mulher para $\mathrm{n}=12$ homens).

Por sua vez, observou-se que nos Comitês Científicos e Corpos de Revisores dos periódicos as assimetrias de gênero são mantidas, conforme apontam os dados da Tabela 9, a seguir. 
Tabela 9. Distribuição por gênero nos comitês científicos e de revisores dos periódicos

\begin{tabular}{c|ccccc}
\hline \multirow{2}{*}{ Periódicos } & \multicolumn{2}{|c|}{ Comitê Científico } & \multicolumn{2}{c}{ Corpo de Revisores } \\
\cline { 2 - 5 } & Mulheres & Homens & Mulheres & Homens \\
\hline$A B C D$ & 1 & 59 & 0 & 0 \\
\hline$A C B$ & 2 & 35 & 0 & 0 \\
\hline$R B C C V$ & 0 & 36 & 0 & 0 \\
\hline$R C B C$ & 0 & 25 & 67 & 5 \\
\hline Total & $\mathbf{3}$ & $\mathbf{1 5 5}$ & $\mathbf{6 7}$ & $\mathbf{5}$ \\
\hline \multicolumn{2}{r}{ Fonte: Elaboração das autoras }
\end{tabular}

Os dados da Tabela 8 mostram que apenas na $R C B C$ - a única revista entre as demais que apresenta uma listagem dos revisores separada da listagem dos membros do Comitê Científico - o total de mulheres $(n=67)$ é muito superior ao total de homens $(n=5)$. Comparado com os dados da Tabela 9, em que não há presença feminina no comitê científico da $\mathrm{RCBC}$, os resultados sugerem que nesse periódico às mulheres é delegada a tarefa de "peer review".

Os resultados exibidos nas Tabelas 8 e 9 corroboram com aqueles obtidos por Kennedy, Lin e Dickstein (2001), que verificou que menos da metade das revistas estudadas tinham paridade entre as percentagens de mulheres nos conselhos editoriais. Dez anos mais tarde, esse cenário parece ter se alterado para pior, considerando a participação feminina nos corpos editoriais, científicos e de revisores nos periódicos da área médica. Dois estudos confirmam essa suposição. O estudo de Heckenberg e Druml (2010) concluiu que ainda persiste um desequilíbrio de gênero nos artigos publicados entre 2001 e 2009 na revista Wiener klinische Wochenschrift: The Middle European Journal of Medicine. Por sua vez, os resultados do estudo de Amrein et al (2011), que investigou a sub-representação feminina nos conselhos editoriais de 60 principais revistas da área de Medicina indexadas na Web of Science, apontaram que apenas em 10 delas os editores-chefes eram do sexo feminino, e menos de um quinto de todos os membros dos corpos editoriais era de mulheres. Concordamos, portanto, com esses autores de que "se mais mulheres fossem nomeadas para atuar em conselhos editoriais isso seria um sinal mais visível de progresso contínuo e serviria como modelo importante para as jovens mulheres que almejam uma carreira acadêmica na Medicina." (AMREIN et al, 2011, p.378).

Frente aos resultados obtidos na pesquisa realizada também concordamos com os argumentos de Cho et al (2014) de que embora as mudanças demográficas na academia tenham reduzido algumas disparidades de gênero ao longo do tempo, as revistas científicas devem ser esforçar de forma proativa visando a paridade de gênero em seus conselhos editoriais. Sem dúvida, no campo da medicina cirúrgica isso poderia contribuir para o incremento de modelos femininos, como também na publicação científica. 


\section{CONSIDERAÇÕES FINAIS}

Os resultados obtidos na pesquisa apontaram que persistem as assimetrias de gênero em termos das autorias, coautorias, tipos de artigos (originais e de revisão), e também no corpo editorial, comitê científico e corpo de revisores, uma vez que nos 920 artigos, escritos por 5.649 autores, houve maior prevalência $(60,8 \%)$ da autoria masculina $(n=3.310)$ em relação à autoria feminina $(n=2.133)$ que obteve $39,2 \%$.

Sugere-se ainda que em futuras pesquisas sobre autoria e coautoria com abordagem bibliométrica também sejam adotados procedimentos que permitam realizar análises qualitativas - tais como o escrutínio da opinião de especialistas - homens e mulheres - a respeito dos padrões de comunicação científica, o que poderá contribuir para oferecer explicações mais aprofundadas a respeito dos aspectos cognitivos envolvidos no processo de comunicação do conhecimento científico. Como afirmam Costas e Bordon (2011), a combinação de indicadores bibliométricos com outras metodologias é relevante, pois permite uma perspectiva mais sociológica nas análises realizadas.

Por último, cabem ainda alguns comentários sobre as dificuldades que os bibliometricistas se deparam ao realizar análises de produção científica, principalmente em estudos que enfocam a questão do gênero de autores e coautores de artigos. É o que acontece, por exemplo, quando se realizam análises de citações e a lista das referências apresentam só as iniciais dos nomes, ou então, quando o artigo é de autoria múltipla apenas o primeiro autor é nomeado e os outros são "et al". Além disso, em várias áreas de conhecimento há periódicos que abaixo do título do artigo utilizam os nomes dos autores abreviados (por exemplo, S. F. Silva) seguidos pelo sobrenome em extenso Em vista disso, vale sugerir aos editores dos periódicos científicos atenção para esses aspectos, para evitar vieses de gênero nas análises da produção científica., A despeito das diferentes normas documentárias adotadas pelos periódicos, uma simples medida a ser tomada nessa direção seria explicitar esses aspectos nas instruções aos autores que visam submeter artigos para publicação

Finalmente, é válido mencionar que embora os resultados aqui descritos não possam ser generalizados, pois apenas quatro periódicos da área de cirurgia foram investigados, a pesquisa realizada contribui para que outras investigações sobre a autoria e coautoria feminina para além da área médica possam ser desenvolvidas, ampliando assim a agenda de pesquisas da área de Ciência da Informação no Brasil. 


\section{CO-AUTORÍA Y PARTICIPACIÓN FEMENINA EN REVISTAS BRASILEÑAS DEL CAMPO DE LA CIRURGÍA:ESTUDIO BIBLIOMÉTRICO}

\section{RESUMEN}

Estudios sobre la co-autoría de las mujeres en la producción científica de varios campos del conocimiento son frecuentes en la literatura científica internacional, pero este objeto de estudio ha sido poco explorado por la investigación brasileña en el campo de las Ciencias Informáticas. Este artículo presenta una contribución a esta area exponiendo los resultados de un estudio que investigó la participación de las mujeres en las co-autorías, y en el cuerpo editorial de revistas científicas brasileñas de la área de la cirugía publicados entre $2010 \mathrm{y}$ 2014. El corpus investigación consistió en 920 artículos publicados en cuatro revistas: Acta Cirúrgica Brasileira (ACB), Arquivos Brasileiros de Cirurgia Digestiva (ABCD), Revista Brasileira de Cirurgia Cardiovascular (RBCCV) y Revista do Colégio Brasileiro de Cirurgiões (RCBC). El enfoque metodológico utilizado fue el análisis bibliométrico y los resultados mostraron que 920 artículos fueron escritos por 5.649 co-autores. Los hombres aparecen como coautores en el 63,5\% $(\mathrm{n}=585)$ de los artículos, mientras que las mujeres aparecen como coautores en el $23,8 \%(\mathrm{n}=219)$ de todos los artículos. Los resultados también mostraron que la participación de las mujeres en las coautorias es menor en los artículos originales y de revisión. El estudio mostró que persisten la desigualdades de género a respeto de las co-autorías, tipos de artículos (originales y revisiones), y también en el Consejo Editorial, el comité científico y el consejo de los colaboradores.

PALABRAS CLAVE: Co-autoría; Bibliometría; Género en la cirugía; Género en la producción científica.

Submetido em: 20/07/2016

Aceito em: 19/12/2016

Publicado em: 19/01/2017

\section{REFERÊNCIAS}

AMREIN, Karin et al. Women underrepresented on editorial boards of 60 major medical journals. Gender Medicine, v.8, n.6, p. 378-387, Dec. 2011.

ÁVILA, Rebeca C. Formação das mulheres nas escolas de medicina. Revista Brasileira de Educação Médica, v.38, n.1, p. 142-149, 2014.

BOURDIEU, Pierre. A dominação masculina. 5.ed. Rio de Janeiro: Bertrand do Brasil, 2007.

CARVALHO, Marília G.; CASAGRANDE, Lindamir S. Mulheres e ciências: desafios e conquistas. Revista Interdisciplinar INTERthesis, Florianópolis, v.8, n.2, p. 20-35, jul./dez. 2011.

$\mathrm{CHO}$, Alyssa $\mathrm{H}$. et al. Women are underrepresented on the editorial boards of journals in environmental biology and natural resource management. PeerJ, v. 2, e542, 2012.

COCHRAN, Amália et al. L. Perceived gender-based barriers to careers in academic surgery.

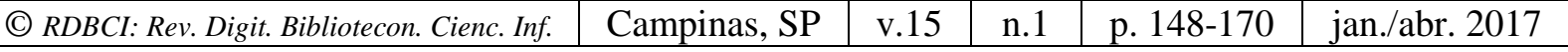


American Journal of Surgery, v.206, p. 263-268.

COSTAS, Rodrigo; BORDÓNS, María. Do age and professional rank influence the order of authorship in scientific publications? Some evidence of micro-level perspective.

Scientometrics, v. 88, p. 145-161, 2011.

EISENBERG, Helen M.; WHALLEY, Elizabeth. Gender and publication patterns: female authorship is increasing, but is there gender parity? Women \& Criminal Justice, v.25, n.1-2, p.130-144, May. 2015.

FASSA, Daniel. Uma vida consagrada: Angelita Habr Gama e sua história de amor e dedicação à medicina. Espaço Aberto, São Paulo, [2007]. Disponível em: <www.usp.br/espacoaberto/arquivo/2007/espaco78abr/ptperfil.htm>. Acesso em: 21 nov. 2014. Acesso em 26 nov. 2014.

FRANCO, Talita; SANTOS, Elizabet G. Mulheres e Cirurgiãs. Revista do Colégio Brasileiro Cirurgiões, v. 37, n. 1, p. 72- 77, 2010.

FRANÇA, Katlein et al. Women in medicine and dermatology: history and advances. Anais Brasileiros de Dermatologia, v.89, n.1, p.182-183, 2014.

FIGUEIREDO, Betânia G. Barbeiros e cirurgiões: atuações dos práticos ao longo do século XIX. História, Ciências, Saúde - Manguinhos, v.6, n.2, p. 277-291, out. 1999.

FITZGERALD, Edward et al. Gender-related perceptions of careers in surgery among new medical graduates: results of a cross-sectional study. The American Journal of Surgery, v.206, p.112-119, 2013.

HARRIS, Linda M.; CHAIKOF, Elliot L.; EIDT, John F. Altering the career choice: can we attract more women to vascular surgery? Journal of Vascular Surgery, v.45, n.4, p. 846848, Apr. 2007.

HAYASHI, Maria Cristina Piumbato Innocentini et al. Indicadores da participação feminina em ciência e tecnologia. Transinformação, v. 19, p.169-187, 2007.

HAYASHI, Maria Cristina Piumbato Innocentini, Hayashi, Carlos Roberto Massao Protocolo para coleta de dados bibliométricos em bases de dados. 2011. (Mimeo).

HECKENBERG, Andrea; DRUML, Christiane. Gender aspects in medical publication: the Wiener Klinische Woscheschrift. Wiener Klinische Woscheschrift, v.122, p. 141-145, 2010.

JAGSI, Reshma et al. The "gender gap" in authorship of academic medical literature - a 35 years perspective. The New England Journal of Medicine, v.355, n.3, p. 281-287, Jul. 2006.

JESUS, Lisilux E. de. Ensinar cirurgia: como e para quem? Revista do Colégio Brasileiro de Cirurgiões, v.35, n.2, p. 136-140, mar./abr. 2008.

JOLLIFF, Lauren; LEADLEY, Jennifer; COAKLEY, Elizabeth. Women in U. S. academic medicine and science: statistics and benchmarking report 2011-2012. Washington:

\begin{tabular}{|c|c|c|c|c|c|}
\hline (C) RDBCI: Rev. Digit. Bibliotecon. Cienc. Inf. & Campinas, SP & v. 15 & n. & p. $148-170$ & jan_/abr. 2017 \\
\hline
\end{tabular}


Association of American Medical Colleges, 2012.

JONASSON, Olga. Leaders in American surgery: where are the women? Surgery, v. 131, n.6, p. 672-675, 2002.

KENNEDY, Barbara L.; LIN, Ying; DICKSTEIN, Leah J. Women in editorial boards of major journals. Academic Medicine, v.76, n.8, p. 849-851, Aug. 2001.

KOSMULSKI, Mareck. The order in the list of authors in multi-author papers revisited. Journal of Informetrics, v. 6, p. 639-644, 2012.

MACHADO, Maria do Céu S. A feminização da medicina. Análise Social, v.38, n.166, p.127-137, 2003.

MALAFAIA, Osvaldo et al. Prevenção do tromboembolismo na cirurgia do câncer do aparelho digestivo. ABCD. Arquivos Brasileiros de Cirurgia Digestiva., São Paulo, v.25, n.4, p. 216-223, out./dez. 2012.

MARINOFF, Lou. O caminho do meio. Rio de Janeiro: Record, 2008.

MARTINS, Mariane D. C. A. et al. Scientific output of Brazilian dermatologists during the last 25 years in the five highest impact factor journals in dermatology. Anais Brasileiros de Dermatologia, v.85, n.5, p. 714-716, 2012.

METAXA, Victoria. Is this (still) a man’s world? Critical Care, v.17, p. 1-3, 2013.

MILLER, Carole A. What's a woman doing in a place like this? Surgical Neurology, v.42, p. 171-176, 1994.

MOREIRA, Herivelto et al. Mulheres pioneiras nas ciências: histórias de conquistas numa cultura de exclusão. In: CONGRESSO IBEROAMERICANO DE CIÊNCIA,

TECNOLOGIA E GÊNERO, 8., 2010, Curitiba. Anais... Curitiba: UTFPR, 2010. p.1-12

MOREIRA JÚNIOR, Dulcídio B.; FIGUEIREDO, Aline; VIEIRA, Camila. Medicina e império: grandes mudanças no panorama científico - de barbeiros a médicos. Revista Alpha, v.13, p.9-19, 2012.

MORTON, Melinda J.; SONNAD, Seema S. Women on professional society and journal editorial boards. Journal of the National Medical Association, v.99, n. 7, p. 764- 771, Jul. 2007.

OLINTO, Gilda. A inclusão das mulheres nas carreiras de ciência e tecnologia no Brasil. Inclusão Social, Brasília, v.5, n.1, p.68-77, jul./dez. 2011.

PARK, Jason et al. Why are women deterred from general surgery? The American Journal of Surgery, v. 190, p. 141-146, 2005.

RISKA, Elianne. Gender and medical careers. Maturitas, v.68, p.264-267, 2011.

\begin{tabular}{|c|c|c|c|c|c|}
\hline (C) RDBCI: Rev. Digit. Bibliotecon. Cienc. Inf. & Campinas, SP & v. 15 & n. & p. $148-170$ & jan./abr. 2017 \\
\hline
\end{tabular}


RIGOLIN, Camila C. D.; HAYASHI, Carlos R. M.; HAYASHI, Maria Cristina P. I. Métricas da participação feminina na ciência e na tecnologia no contexto dos INCTs: primeiras aproximações. Liinc em Revista, v. 9, p. 143-170, 2013.

SANFEY, Hilary A. Influences on medical student career choice. Archives of Surgery, v.141, p. 1086-1095, Nov. 2006.

SANTOS, Tania S. Divisão sexual do trabalho na profissão médica e atividades acadêmicas. In: Congresso Iberoamericano de Ciência, Tecnologia e Gênero, 8., 2010, Curitiba. Anais... Curitiba: UTFPR, 2010. Disponível em:

<http://files.dirppg.ct.utfpr.edu.br/ppgte/eventos/cictg/conteudo_cd/artigos_7.html>Acesso em 26 nov. 2014.

SCHIEBINGER, Londa. O feminismo mudou a ciência? Bauru: EDUSC, 2001.

SILVA, Márcia Regina da.; HAYASHI, Carlos R. M.; HAYASHI, Maria Cristina Piumbato Innocentini. Análise bibliométrica e cientométrica: desafios para especialistas que atuam no campo. InCID: Revista de Ciência da Informação e Documentação, v. 2, p. 110-129, 2011.

WEINACKER, Ann; STAPLETON, Renne. Still a man's world, but why? Critical Care, v.17, p. 1-2, 2013.

WEST, Jevin D.; JACQUET, Jennifer; KING, Molly; CORREL, Shelley J.; BERGSTROM, Carl T. The role of gender in scholarly authorship. PLOS One, v.8, n.7, e66212, Jul. 2013.

ZENG, Xiao Han; DUCH, Jordi; SALES-PARDO, Marta; MOREIRA, João A. G.;

RADICCHI, Filippo; RIBEIRO, Haroldo V.; WOODRUFF, Teresa K.; AMARAL, Luís A. Nunes. Differences in collaboration patterns across disciplines, career stage, and gender.

PLOS Bio, v.14, n.1, e1002573, Nov. 2016

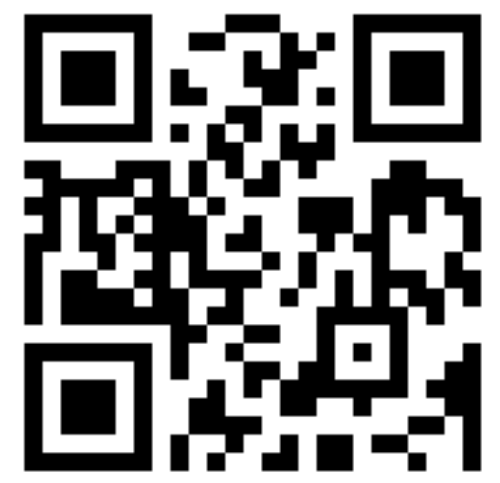

